hep-th/0311122

CPHT-RR 106.1103

\title{
Mirror symmetric SU(3)-structure manifolds with NS fluxes
}

\author{
Stéphane Fidanza, Ruben Minasian and Alessandro Tomasiello \\ Centre de Physique Théorique, Ecole Polytechnique \\ Unité mixte du CNRS et de l'EP, UMR 7644 \\ 91128 Palaiseau Cedex, France
}

\begin{abstract}
When string theory is compactified on a six-dimensional manifold with a nontrivial NS flux turned on, mirror symmetry exchanges the flux with a purely geometrical composite NS form associated with lack of integrability of the complex structure on the mirror side. Considering a general class of $T^{3}$-fibered geometries admitting SU(3) structure, we find an exchange of pure spinors $\left(e^{i J}\right.$ and $\Omega$ ) in dual geometries under fiberwise $\mathrm{T}$-duality, and study the transformations of the NS flux and the components of intrinsic torsion. A complementary study of action of twisted covariant derivatives on invariant spinors allows to extend our results to generic geometries and formulate a proposal for mirror symmetry in compactifications with NS flux.
\end{abstract}

October 9, 2018 


\section{Introduction}

Mirror symmetry is a pairing between different compactifications which give rise to the same fourdimensional effective theory. For Calabi-Yau compactifications it is well-understood and has played an important role, becoming arguably the most interesting mathematical application of string theory. More general compactifications with fluxes on manifolds which are not Ricci-flat have become focus of much attention recently, and it would be important to extend to these at least partially the machinery which proved so useful for Calabi-Yaus.

If we had to consider only supersymmetric vacua, our search would be premature. The conditions on fluxes and warping to compensate non-Ricci-flatness and preserve supersymmetry are wellunderstood for some types of fluxes. To some extent, as we review later, these conditions are even translated into mathematical requirements: the manifold has to have $\mathrm{SU}(3)$ structure and fall into a certain class in the mathematical classification of these objects. But Bianchi identity becomes an equation for which there is no existence theorem in the literature, unlike the famous Yau's theorem for Calabi-Yaus (not even the analogue of Calabi conjecture seems to have been formulated: this might be a task for string theory). If there is no singularity in the internal compact manifold, and the higher derivative terms are not taken into account, one can actually show even non-existence theorems.

Fortunately mirror symmetry as a more general equivalence of effective theories, and not only of vacua, still makes sense. As emphasized in [1], to have supersymmetry of the effective action $\mathrm{SU}(3)$ structure is enough, without the extra requirements mentioned above, which ensure we are actually in a supersymmetric vacuum. Not only looking for mirror symmetric SU(3) manifolds makes sense, but it is sensible to expect that a formal advance in this direction might help to understand the still elusive problem of compactifications with fluxes.

Much in this spirit, 1] (building on a comment in 2]) considered a particular case. Namely, an $H$ flux on Calabi-Yau manifolds (without back-reaction: we are not dealing with a vacuum) get mapped to so-called half-flat manifolds, a particular class of SU(3) structure manifolds, without any $H$ flux. The amount by which these half-flat manifolds fail to be Ricci-flat is measured by a certain quantity called intrinsic torsion. We can thus say that, in this example, $H$ flux gets exchanged by mirror symmetry with components of the intrinsic torsion associated with lack of integrability of the complex structure.

It is natural to wonder what happens in more general cases, when on both sides one has both $H$ and intrinsic torsion. (As mentioned above, this for example is necessary in order to have supersymmetric vacua.) In the Calabi-Yau case, a concrete approach to mirror symmetry is StromingerYau-Zaslow (SYZ) 3] conjecture. This states that $i$ ) every Calabi-Yau is a $T^{3}$ special lagrangian fibration over a three-dimensional base, and ii) mirror symmetry is T-duality along the three circles of the $T^{3}$. It is natural to try and generalize this method to the present problem. Part $i$ ) of the conjecture came originally from considering moduli spaces of D-branes on Calabi-Yaus; generalizing this to background with fluxes does seem premature, and in any case we do not attempt it here, although later we will comment more on it. So we simply assume the manifold and flux we start with have this property, of admitting three Killing vectors. The idea is that the mirror transformations found in this class of examples will generalize to some extent to the most general case.

Having assumed this, we perform $\mathrm{T}$-duality along the three isometries at once. $\mathrm{T}$-duality will preserve four-dimensional effective theories, but since eventually we hope this procedure could be extended to more general situations by including singular fibers as in SYZ, we want to show why this should be called mirror symmetry - for that matter, indeed, why is there any mirror symmetry at all. A good framework for answering this is Hitchin's method based on Clifford(6,6) spinors [4]. As we review later in more detail, these are simply formal sums of forms on the manifold. Existence on a manifold of a Clifford $(6,6)$ spinor without zeros which is also pure (annihilated by half of the gamma matrices) is the same as saying that there is a $\mathrm{SU}(3,3)$ structure on the manifold. (If the spinor is also closed, Hitchin calls these manifolds generalized Calabi-Yaus.) For a $\mathrm{SU}(3)$ structure, 
there are two pure spinors which are orthogonal and of unit norm. From this point of view it is natural to conjecture that mirror symmetry between two $\mathrm{SU}(3)$ structure manifolds exchanges these two pure spinors. We can be more explicit if we compare this Clifford $(6,6)$ spinor definition of $\mathrm{SU}(3)$ structure with the more usual one, existence of a two-form $J$ and three-form $\Omega$ obeying $J \wedge \Omega=0$ and $i \Omega \wedge \bar{\Omega}=(2 J)^{3} / 3$ !. In these terms the two pure spinors are $e^{i J}$ and $\Omega$. We can actually multiply first spinor by $e^{B}$ leaving it pure 4 . So what we are claiming is

$$
e^{B+i J} \longleftrightarrow \Omega
$$

The arrows here will be made precise in section 3 In the Calabi-Yau case, this exchange is implicit in many applications of mirror symmetry. For example, the even periods and the D-brane charge can be written using $e^{B+i J}$, and its exchange with $\Omega$ was used in mapping [5] stringy-corrected DUY equations [6] to the special lagrangian condition; $e^{B+i J}$ was also used in formulating the concept of $\Pi$-stability [7].

With this in mind, we check that $\mathrm{T}$-duality along $T^{3}$ (when it is possible) realizes the exchange (1.1), and for this reason we call it mirror symmetry. In this sense we have generalized part ii) of SYZ. However as it stands, (1.1) is hardly useful in predicting the mirror background starting from a particular six-manifold and NS flux.

After having discussed and justified the method, we can schematically describe here our results. The usual quantities which measure non-Ricci-flatness of the SU(3) manifold are the five components of the intrinsic torsion (mentioned above) labeled as $W_{i}, i=1 \ldots 5$, in the representations $1 \oplus 1,8 \oplus 8,6 \oplus \overline{6}, 3 \oplus \overline{3}, 3 \oplus \overline{3}$ respectively. What is puzzling at first is that one does not see many ways of mirror pairing these representations, except for $W_{4}$ and $W_{5}$ which are two vectors. The answer is that the two mirrors have indeed two different $\mathrm{SU}(3)$ structures: the two $\mathrm{SU}(3)$ are differently embedded into $\operatorname{Spin}(6,6)$, because the fiber directions change from tangent bundle to cotangent bundle, roughly speaking. As a result, representations get actually mixed. What is preserved is the representations that these objects have once pulled back to the base manifold, which is untouched by T-duality. $W_{2}$ and $W_{3}$ get then split as $W_{2}=w_{2}^{s}+w_{2}^{a}(8 \rightarrow 5 \oplus 3)$ and $W_{3}=w_{3}^{s}+w_{3}^{t}$ $(6 \rightarrow 5 \oplus 1)$, and we get

$$
\begin{gathered}
W_{1}-i H_{1} \longleftrightarrow-\overline{\left(W_{1}-i H_{1}\right)}, \\
\bar{w}_{2}^{s} \longleftrightarrow w_{3}^{s}-i h_{3}^{s}, \\
w_{5}, \bar{w}_{2}^{a} \longleftrightarrow w_{4}-i h_{4} .
\end{gathered}
$$

A more detailed discussion of these equations can be found in section 4 (see in particular (4.11) for the precise statement). In (1.2) one can see that $W_{1}, W_{3}, W_{4}$ get naturally complexified by the components of $H$ in the corresponding representation. This is no surprise as these torsions appear, as we review later, in $d J$, and the natural object in string theory is always $B+i J$. In the present context, this arises rather due to the usual combination $E=g+B$ of T-duality. As we will specify in section 2 we mostly work with a purely base-fiber type $B$-field, which is not the most general form allowed by $T^{3}$ invariance. However, we will see that this is just a simplifying technical assumption, and may eventually be relaxed. Note also that (1.2) complements (1.1) in an essential way by specifying in a more practical fashion the data of the mirror background (the metric and the NS flux). In particular, it quantifies the exchange of components of the flux and the intrinsic torsion on mirror sides.

We have also indicated in (1.2) that some of the components of the intrinsic torsion we begin with are actually related; so $T^{3}$ fibrations are not the most general $\mathrm{SU}(3)$ manifold. This in a way answers in the negative the question about generalizing part $i$ ) of SYZ: we are getting mirror symmetry only for a subclass of manifolds. In particular, supersymmetric vacua with only the three-form switched on are outside this class: indeed the conditions for these are [8] (reinterpreted in terms of torsions for example in [9] and [10])

$$
W_{1}=W_{2}=0, \quad W_{3}=* H_{3}, \quad W_{4}=d \phi=i H_{4}, \quad W_{5}=2 d \phi
$$


where we denoted by $H_{4}$ and $H_{3}$ the components in the representations 3 and 6 of $\mathrm{SU}(3)$ respectively, in analogy with the notation for torsions (see also the appendix @). So the next natural step would be to try and include more general classes, among which maybe supersymmetric vacua ${ }^{1}$. In order to do so, it is natural to wonder to what extent the transformation rules can be put in a nicer form, and in particular be covariantized. It turns out that it is convenient to use spinors. Although also the previously mentioned Clifford $(6,6)$ spinors can be used, here we mean a more conventional Clifford(6) spinor without zeros. One such spinor, call it $\epsilon$, always exists on any SU(3) structure manifold and can actually be used to define it. It turns out that using $\epsilon$ a different basis for $W$ 's can be defined, which is diagonal under T-duality: elements of the basis transform picking a sign.

The idea of this different spinorial basis for $W$ 's is roughly speaking the following. Usual $W$ 's are defined, as we review later, from $d J$ and $d \Omega$. Now, not only $\epsilon$ is equivalent to the pair $J, \Omega$, but the information contained in $d J$ and $d \Omega$ can also be completely extracted from $D_{M} \epsilon$. Using $\mathrm{SU}(3)$ structure, this can be decomposed as $D_{M} \epsilon=\left(q_{M}+i \tilde{q}_{M} \gamma+i q_{M N} \gamma^{N}\right) \epsilon$, where $\gamma$ is the chiral gamma in six dimensions and the group representations inside the quantities $q_{M}, \tilde{q}_{M}, q_{M N}$ are in one-to-one correspondence with the $W$ 's. Switching to the spinorial basis accomplishes two things. First, it allows to capture the exchange of the pure spinors $e^{i J}$ and $\Omega$ and the exchange of their integrability properties simultaneously. More importantly, it allows to conjecture the sixdimensional covariantization of the mirror transformation (1.2), written in terms of the forms pulled back to the base of the $T^{3}$ fibration. Details can be found in section 5

For the purposes of studying mirror symmetry/T-duality we will need first to introduce the covariant derivative twisted by the NS flux:

$$
D_{M}^{H} \epsilon=\left(Q_{M}+i \tilde{Q}_{M} \gamma+i Q_{M N} \gamma^{N}\right) \epsilon
$$

where, as we will see in detail, $Q$ 's are obtained from $q$ 's by complexifying certain components of the intrinsic torsion by the matching components of the flux (as in (1.2)). We will show that their restrictions to the base (denoted by hatted quantities) transform as

$$
\hat{Q}_{i j} \longrightarrow-\overline{\hat{Q}}_{i j}, \quad \hat{Q}_{i} \longrightarrow-\overline{\hat{Q}}_{i} .
$$

We will then argue that this simplification is due to the simple transformation of the ten-dimensional spinors under T-duality.

Finally we will try, in section [5.1 to collect these several points of view to argue that in general a rule like

$$
Q_{m n} \longleftrightarrow-Q_{m \bar{n}}, \quad Q_{m} \longleftrightarrow-\bar{Q}_{m}
$$

should hold. This rule is consistent with what we found in the $T^{3}$ fibered case, and with the principle that supersymmetric vacua should map in supersymmetric vacua (not necessarily the same). There are however more checks that could be done if one understood better examples; we discuss this in section 6. For example, in the case we mentioned above of compactifications with $H$ only described by (1.3), one should understand moduli spaces and then check that a kind of exchange of complex and Kähler moduli (although, as we will argue, this has to be taken with a grain of salt) should happen. This might be interesting for the problem of fixing moduli. We end with a section on open problems.

\section{Geometric setting}

We start with an introductory section on T-duality, mainly to fix the notations.

The six-dimensional manifold will be taken to be a $T^{3}$ fibration over a base $B$. Coordinates on the base will be denoted by $\left(y^{1}, y^{2}, y^{3}\right)$, and on the fiber by $\left(x^{1}, x^{2}, x^{3}\right)$. All the quantities will only depend on the $y$ coordinates, so that the $x$ directions are Killing vectors. We will use conventions for indices as follows:

\footnotetext{
${ }^{1}$ There may actually be supersymmetric vacua involving $T^{3}$ fibrations, if other fields are included.
} 
- $i, j, k, \ldots$ are used in the $3 \mathrm{~d} y$ subspace,

- $\alpha, \beta, \gamma, \ldots$ are used in the $3 \mathrm{~d} x$ subspace,

- $M, N, \ldots$ are used in the total $6 \mathrm{~d}$ space for real coordinates : $d y^{M}=\left(d y^{i}, d x^{\alpha}\right)$,

- $m, n, \ldots$ are used for holomorphic/antiholomorphic indices,

- $A, B, C, \ldots$ are indices in the total $3 \mathrm{~d}$ complex frame space,

- $a, b, c, \ldots$ and $a^{\prime}, b^{\prime}, c^{\prime}, \ldots$ are used in the $3 \mathrm{~d}$ real $y$ and $x$ frame spaces. Primes will be dropped quickly.

We write then the most general metric and B field as ${ }^{2}$

$$
\begin{aligned}
d s^{2} & =g_{i j} d y^{i} d y^{j}+h_{\alpha \beta} e^{\alpha} e^{\beta}=G_{M N} d y^{M} d y^{N} \\
B_{2} & =\frac{1}{2} B_{i j} d y^{i} \wedge d y^{j}+B_{\alpha} \wedge\left(d x^{\alpha}+\frac{1}{2} \lambda^{\alpha}\right)+\frac{1}{2} B_{\alpha \beta} e^{\alpha} \wedge e^{\beta}
\end{aligned}
$$

where $\lambda^{\alpha}=\lambda_{i}^{\alpha} d y^{i}, B_{\alpha}=B_{i \alpha} d y^{i}$ and we have defined

$$
e^{\alpha} \equiv d x^{\alpha}+\lambda^{\alpha}
$$

Of course the vielbein reads $\left(e_{i}^{a} d y^{i}, V_{\alpha}^{a} e^{\alpha}\right)$, where

$$
\begin{array}{ll}
\delta_{a b} e_{i}^{a} e_{j}^{b}=g_{i j}, & \delta_{a^{\prime} b^{\prime}} V_{\alpha}^{a^{\prime}} V_{\beta}^{b^{\prime}}=h_{\alpha \beta}, \\
g^{i j} e_{i}^{a} e_{j}^{b}=\delta^{a b}, & h^{\alpha \beta} V_{\alpha}^{a^{\prime}} V_{\beta}^{b^{\prime}}=\delta^{a^{\prime} b^{\prime}} ;
\end{array}
$$

we also record that the inverse vielbein has instead the form

$$
e_{a}^{i}\left(\frac{\partial}{\partial y^{i}}-\lambda_{i}^{\alpha} \frac{\partial}{\partial x^{\alpha}}\right), \quad V_{a}^{\alpha} \frac{\partial}{\partial x^{\alpha}} .
$$

T-duality along the three $x^{\alpha}$ directions can be expressed conveniently in terms of the quantity $E=g+B$

$$
\begin{aligned}
& E_{i j} d y^{i} d y^{j}+E_{i \alpha} d y^{i} d x^{\alpha}+E_{\alpha i} d x^{\alpha} d y^{i}+E_{\alpha \beta} d x^{\alpha} d x^{\beta} \\
& \mapsto E_{i j} d y^{i} d y^{j}+E^{\alpha \beta}\left(d x^{\alpha}+E_{i \alpha} d y^{i}\right)\left(d x^{\beta}+E_{\beta j} d y^{j}\right) ;
\end{aligned}
$$

notice that in this expression all the (implicit) tensor products are neither symmetrized nor antisymmetrized, for example $d y^{i} d y^{j}=d y^{i} \otimes d y^{j}$. Also remark that in this expression we used $d y^{i}, d x^{\alpha}$ basis instead of $d y^{i}, e^{\alpha}$ as virtually everywhere else. $E^{\alpha \beta}$ is the inverse of $E_{\alpha \beta}$ and can be decomposed in symmetric and antisymmetric part:

$$
E^{\alpha \beta}=\left(\frac{1}{h+B}\right)^{\alpha \beta}=\hat{h}^{\alpha \beta}+\hat{B}^{\alpha \beta} \quad \text { where }\left\{\begin{aligned}
\hat{h} & =\frac{1}{h+B} h \frac{1}{h-B} \\
\hat{B} & =\frac{1}{h+B}(-B) \frac{1}{h-B}
\end{aligned}\right.
$$

The objects $\hat{h}$ and $\hat{B}$ would also called in other contexts $H$ and $\Theta$. (In this paper $H$ denotes instead the three-form field.)

Using the relations

$$
\hat{B} \hat{h}^{-1}=-h^{-1} B ; \quad \hat{h}^{-1} \hat{B}=-B h^{-1} ; \quad \hat{B} \hat{h}^{-1} \hat{B}=\hat{h}-h^{-1}
$$

\footnotetext{
${ }^{2}$ with the convention that $\omega_{1} \wedge \omega_{2}=\omega_{1} \otimes \omega_{2}-\omega_{2} \otimes \omega_{1}$
} 
one can show that the T-dual metric and $B$ field can be obtained by the original ones (2.1), (2.2) by the substitutions

$$
h_{\alpha \beta} \longleftrightarrow \hat{h}^{\alpha \beta} ; \quad B_{\alpha \beta} \longleftrightarrow \hat{B}^{\alpha \beta} ; \quad B_{\alpha} \longleftrightarrow \lambda^{\alpha}
$$

and leaving the $g_{i j}$ and $B_{i j}$ in variant. Notice that last equation in (2.6) means that the twisting of each of the three $S^{1}$ bundles gets exchanged with $B$ field. This fact played for example a role in a number of applications and was recently formalized in mathematical terms in 11 .

We can also find the vielbein $\hat{V}^{a \alpha}$ of the T-dual metric $\hat{h}^{\alpha \beta}$, that satisfies $\hat{V}^{a \alpha} \hat{V}^{a \beta}=\hat{h}^{\alpha \beta}$ :

$$
\hat{V}^{a \alpha}=\left(\frac{1}{h+B}\right)^{\alpha \beta} V_{\beta}^{a}=V_{\beta}^{a}\left(\frac{1}{h-B}\right)^{\beta \alpha}
$$

whose inverse is

$$
\hat{V}_{\alpha}^{a} \equiv \hat{h}^{\alpha \beta} \hat{V}^{a \beta}=(h-B)_{\alpha \beta} V^{a \beta}=V^{a \beta}(h+B)_{\beta \alpha} .
$$

The T-duality transformations of the vielbeine then are:

$$
V_{\alpha}^{a} \longleftrightarrow \hat{V}^{a \alpha} ; \quad V^{a \alpha} \longleftrightarrow \hat{V}_{\alpha}^{a} .
$$

We will mostly work in the case when the $B$-field is purely of base-fiber type in frame indices. Transformation (2.6) shows that this condition is conserved by T-duality, while (2.5) reduces to $\hat{h}^{\alpha \beta}=h^{\alpha \beta}$. Consequently, $\hat{V}^{a \alpha}=V^{a \alpha}$ and $\hat{V}_{\alpha}^{a}=V_{\alpha}^{a}$. T-duality then only amounts to moving fiber indices up and down (still exchanging $B_{\alpha}$ and $\lambda^{\alpha}$ though).

For later use, we also define here the tensors defining the SU(3) structure. These would be a priori only a two-form $J$ and a three-form $\Omega$ satisfying $J \wedge \Omega=0$ and $i \Omega \wedge \bar{\Omega}=(2 J)^{3} / 3$ !, but here we define the structure in a more conventional way starting from an almost complex structure. The latter is defined by giving the $(1,0)$ vielbein

$$
E^{A}=i e_{i}^{a} d y^{i}+V_{\alpha}^{a^{\prime}} e^{\alpha}
$$

where $A=a=a^{\prime}$ goes from 1 to 3 . The corresponding $(0,1)$ vielbein is $E^{\bar{B}}=\overline{E^{B}}$. This almost complex structure is in general not integrable, as (even after rescaling) it is not expressible as $d$ of a complex coordinate, $E^{A} \neq \alpha^{A} d z^{A}$. However, with an abuse of language we will use the quantity

$$
d z^{j} \equiv d y^{j}-i V_{\gamma}^{j} e^{\gamma}=-i e_{a}^{j} E^{a}
$$

keeping in mind that there is no reason for an actual coordinate $z^{i}$ to exist. We also used in this expression the notation

$$
V_{i \alpha} \equiv \delta_{a a^{\prime}} e_{i}^{a} V_{\alpha}^{a^{\prime}}=e_{i}^{a} V_{a \alpha}
$$

The two-form $J$ (sometimes called fundamental form) is defined by

$$
J=\frac{i}{2} \delta_{A B} E^{A} \wedge E^{\bar{B}}=\frac{i}{2} g_{i j} d z^{i} \wedge d \bar{z}^{j}=-V_{i \alpha} d y^{i} \wedge e^{\alpha}
$$

The holomorphic 3-form reads instead

$$
\Omega=E^{1} \wedge E^{2} \wedge E^{3}=\frac{1}{6} \epsilon_{A B C} E^{A} \wedge E^{B} \wedge E^{C}=-\frac{i}{6} \epsilon_{i j k} d z^{i} \wedge d z^{j} \wedge d z^{k},
$$

where $\epsilon^{i j k}=\epsilon_{a b c} e^{a i} e^{b j} e^{c k}$.

The choices we are making for the SU(3) structure are inspired by the SYZ approach. As we stressed above, these choices reduce the structure group further and are thus not to be expected to be as general (not even locally) as the $T^{3}$ fibration structure was in the SYZ approach. In particular some unaesthetic features will arise later in the dual of the complex coordinates. Anyway, in section 5.1 we will try to amend to this loss of generality. 


\section{Mirror symmetry as $\mathbf{T}$-duality}

We start in this section showing, as promised in the introduction, how $e^{B+i J}$ and $\Omega$ get exchanged by T-duality.

First we do the easier case, in which there is no $B$ field and $\lambda$ twisting of the $T^{3}$ bundle. The basic idea is that $\Omega$ can be written in a sense as an exponential of the almost complex structure $J_{M}{ }^{N}$ applied to a degenerate three-form $\epsilon_{i j k} d y^{i} d y^{j} d y^{k}$, that can be thought of as the holomorphic three-form in the large complex structure limit. A way to be more explicit is the following. Expand $\Omega$ from (2.13) using the expression for the holomorphic vielbein in (2.10). One obtains four terms, with $d y^{3}, d y^{2} e$, and so on. Define now the operation $\left.V^{\perp}\right\lrcorner(\cdot)$ by

$$
\left.V^{\perp}\right\lrcorner\left(e^{\alpha_{1}} \ldots e^{\alpha_{k}}\right)=\frac{1}{(3-k) !} \epsilon^{\alpha_{1} \ldots \alpha_{3}} e_{\alpha_{k+1}} \ldots e_{\alpha_{3}}, k=0 \ldots 3 .
$$

This is essentially a Hodge star on the fiber, except it sends a $k$-form in the fiber into a $3-k$-vector (a section of $\Lambda^{3-k} T$ ). Lower $e_{\alpha}$ are indeed vectors $\partial_{\alpha} \equiv \partial / \partial x^{\alpha}$. This operation is very similar to the T-duality transformation of spinors to be discussed shortly. Using this, on every component of the expansion in $d y$ and $e$ of $\Omega$, we get a sum of $(k, k)$ tensors, namely $k$ indices up and $k$ down: those down are along the fiber. The sum can be expressed as an exponent of $V_{i}^{\alpha} e_{\alpha} d y^{i}$, which is the complex structure. T-duality is now easy to perform. According to (2.9) its action is simply to raise and lower $\alpha$ index: the tangent bundle (in the fiber direction) of the starting manifold is equal to the cotangent bundle (again in the fiber direction) of the T-dual manifold. As a result the complex structure gets now mapped to $V_{i \alpha} e^{\alpha} d y^{i}$, the fundamental two-form $J$. So we have gotten

$$
\left.T\left(V^{\perp}\right\lrcorner \Omega\right)=\frac{i}{3 !} e^{i J} .
$$

The case with $B$-field and $\lambda$ is less trivial. Although this is not strictly required here, we find already at this point helpful to think about this in terms of Clifford(d,d) spinors. So we make a brief intermezzo explaining these and then we get back to our computation. Much of this material is taken from [4.

\subsection{Clifford(d, d) spinors}

Clifford algebra is usually defined on the tangent bundle (or cotangent) of a manifold using the metric. In physical notation this amounts to defining $d$ gamma matrices which satisfy $\left\{\gamma^{M}, \gamma^{N}\right\}=$ $2 g^{M N}$, where $g^{M N}$ is the metric on the cotangent bundle of the manifold. On SU(3) manifold there is moreover a well-known representation of this Clifford algebra, on $\Omega^{0, p}$ forms. If we on the contrary forget about the metric (thus about the $\mathrm{SO}(\mathrm{d})$ structure), this algebra cannot be defined.

If we consider, however, both the tangent and the cotangent bundles of the manifold at the same time, there is a natural pairing between them (namely contraction between a vector and a form, $\left.\left(d y^{M}, \partial_{N}\right)=\delta^{M}{ }_{N}\right)$, in which the metric does not enter. This "metric" on $T \oplus T^{*}$ is block-offdiagonal

$$
\left(\begin{array}{ll}
0 & 1 \\
1 & 0
\end{array}\right)
$$

and thus of signature $(d, d)$. Concretely, what this means is that one has to define $2 d$ independent gamma matrices, $\gamma^{M}, \gamma_{M}$, that satisfy $\left\{\gamma^{M}, \gamma^{N}\right\}=0=\left\{\gamma_{M}, \gamma_{N}\right\}$ and $\left\{\gamma^{M}, \gamma_{N}\right\}=\delta_{N}^{M}$. Even though the Clifford structure has been defined on $T \oplus T^{*}$, fortunately the algebra still has a representation in terms of the forms on the manifold. Only now we have twice the number of creators and annihilators, and instead of using simply $(0, p)$ forms as before, we have to use forms of all possible degrees. On this space $\oplus_{p=1}^{d} \Lambda^{p} T^{*}$, an explicit representation is

$$
\gamma^{M}=d y^{M} \wedge, \quad \gamma_{M}=\iota_{\partial_{M}} .
$$


In all this we stress again that we have to consider $\gamma_{M}$ and $\gamma^{M}$ as independent: we cannot raise and lower indices using the metric. In this Clifford(d,d) algebra, however, the usual Clifford(d) is embedded: indeed a combination of wedge and contraction in (3.2) is the more conventional Clifford product, and if we use that we can raise and lower indices.

As stated in the introduction, a pure spinor is one which is annihilated exactly by half of the gamma matrices. If we come back at the application we have in mind, both $e^{i J}$ and $\bar{\Omega}$ are pure:

$$
\left(\gamma^{M}-i J^{M N} \gamma_{N}\right) e^{i J}=0, \quad \begin{aligned}
& \left(\gamma^{M}-i J^{M}{ }_{N} \gamma^{N}\right) \bar{\Omega}=0 \\
& \left(\gamma_{M}-i J_{M}{ }^{N} \gamma_{N}\right) \bar{\Omega}=0 .
\end{aligned}
$$

The gammas that annihilate the pure spinor $\bar{\Omega}$ are more familiar if one expresses them in holomorphic/antiholomorphic indices: $\gamma^{m} \Omega=\gamma_{\bar{m}} \Omega=0$. Indeed $\bar{\Omega}$ is one of the Clifford vacua for the Clifford(d) representation mentioned above (this is why we wrote the relations for $\bar{\Omega}$ rather than $\Omega$ ). Let us also notice that the annihilators of the two Clifford(d, d) spinors in (3.3) become the same when we allow ourselves to raise and lower indices on gammas, that is, when we descend to Clifford(d): $e^{i J}$ becomes then an alternative expression for a Clifford vacuum of Clifford(d).

As already mentioned, this dual way of realizing Clifford(d) from Clifford(d, d) is obviously in the center of mirror symmetry - exchange of the the Kähler form and the holomorphic three-form (or their non-integrable generalizations) is seen as different choices of Clifford vacuum.

\subsection{Back to mirror symmetry}

In this section, the only parts of the above theory that we actually use here are the formulas for the annihilators (3.3), which of course could have been derived independently. This insight gives however a useful rule of thumb, in particular when dealing with $e^{i J}$, where we can save ourselves expanding the exponential as we did above. What we will do in the following will be to consider $e^{i J}$ and $\Omega$ as Clifford(6,6) spinors, and other forms acting on them as combinations of gamma matrices. This is of course not the only possibility. One might have included $B$ in the definition of the pure spinor. Due to technical details in how T-duality works we preferred this way. Also, we will work here in the case $B_{\alpha \beta}=0$.

Let us consider for example the expression $e^{B} \Omega$. Due to $\gamma^{\alpha} \Omega=i \gamma^{i} V_{i}^{\alpha} \Omega$, this equals $e^{i B_{\alpha} \wedge V^{\alpha}} \Omega$. If we act on this with the operator $\left.V^{\perp}\right\lrcorner$ defined above, the prefactor can be taken out (it does not contain any $\left.e^{\alpha}\right)$. On $\Omega$ we get $\left.V^{\perp}\right\lrcorner(\Omega)=e^{-i V^{\alpha} e_{\alpha}}$ as above; the only thing to notice is that $e_{\alpha}$ is simply $\partial_{\alpha}$, as seen on (2.3). If we finally apply T-duality, $V^{\alpha} e_{\alpha} \mapsto V_{\alpha} e^{\alpha}$; putting it together with the inert factor $e^{i B_{\alpha} \wedge V^{\alpha}}=e^{i B^{\alpha} \wedge V_{\alpha}}$, we have shown

$$
\left.\frac{i}{3 !} T\left(e^{i J}\right)=V^{\perp}\right\lrcorner\left(e^{B} \Omega\right) e^{-B_{\alpha} \lambda^{\alpha}} .
$$

It is a little surprising that the $B$ field has to be subtracted on right hand side rather than being already present on left hand side. In the same way we can also prove the more reassuring

$$
\left.T(\Omega)=\frac{i}{3 !} V^{\perp}\right\lrcorner\left(e^{B} e^{i J}\right) e^{B_{\alpha} \lambda^{\alpha}} .
$$

The exchange $e^{B+i J} \longleftrightarrow \Omega$ as presented in (3.4) and (3.5) is not very aesthetically pleasing, however the exponents involving the T-duality anti-invariant $B_{\alpha} \lambda^{\alpha}$ are easy to explain going back to (3.3). The condition of purity e.g. on $\Omega$ is essentially $d z \wedge \Omega=0$, and the holomorphic coordinates change under T-duality. The reason of this is that the $d z^{i}$ which we have defined above as $d y^{i}-i V_{\gamma}^{i} e^{\gamma}$ has a $\lambda$ hidden inside $e^{\gamma}$. Since $\lambda$ gets exchanged with $B$ due to (2.6), $d z$ on the original manifold does not map exactly to $d z$, but $d z \longrightarrow d z-i\left(B_{\alpha} V^{\alpha}-\lambda^{\alpha} V_{\alpha}\right)$ shifting by another T-duality antiinvariant. Thus the role of $e^{ \pm B_{\alpha} \lambda^{\alpha}}$ is to compensate for this change, preserving the condition for purity. 
The combinations $e^{i J}$ and $\Omega$ allow, as we have commented on in the introduction and as we will see further later on, to treat $J$ and $\Omega$ more symmetrically. The most symmetrical object one might imagine is actually the $\mathrm{SU}(3)$ invariant spinor $\epsilon$ itself. Given also the role that we anticipated it will have in torsions, one might wonder at this point if it is more convenient to use T-duality transformation of $\epsilon$ and forget all the rest. The problem is, so to say, that the spinor is too symmetric. The transformation rule of the ten-dimensional spinors are known: in the case without $B_{\alpha \beta}$, we simply have $\psi_{+} \rightarrow \psi_{+}, \psi_{-} \rightarrow \gamma_{f} \psi_{-}$, where $\gamma_{f}$ is the product of the three gammas in the fiber directions [12]. However, when we express $\psi_{ \pm}$in terms of the chirality projected $\epsilon_{ \pm}$of the sixdimensional spinor, $\gamma_{f} \epsilon_{+}$is actually $\epsilon_{-}$and all the information we get is that a IIA compactification has been exchanged with a IIB one. This means that the spinor is essentially on both the original and the T-dual manifold the pull-back of a spinor in the base. Still, using the familiar bilinear definitions for $J$ and $\Omega$ (5.2) and $\gamma_{f} \epsilon_{+}=\epsilon_{-}$, one can show the identities above in a different way.

\section{Intrinsic torsions and their duals}

This section is the technical core of the paper. Here we define and compute intrinsic torsions for our $T^{3}$ fibered manifolds. As stressed in the introduction, these are not the most general SU(3) structure manifolds. Performing T-duality along the $T^{3}$ is then easy using (2.6) and (2.9).

\subsection{Conventional definition of torsions}

We do not aim here at reviewing intrinsic torsions on manifolds with G-structures as discussions already exist in the literature, see for example [13] and among recent physics papers 1, 9]. Here we give a good working definition. It is familiar that, if we are on a SU(3) holonomy manifold, not only $J$ and $\Omega$ are well defined, but also they are closed: $d J=0=d \Omega$. If they are not, $d J$ and $d \Omega$ give a good measure of how far the manifold is from having $\mathrm{SU}(3)$ holonomy. The usual definitions require to split them in $\mathrm{SU}(3)$ representations:

$$
\begin{gathered}
d J=-\frac{3}{2} \operatorname{Im}\left(W_{1} \bar{\Omega}\right)+W_{4} \wedge J+W_{3} \\
d \Omega=W_{1} J^{2}+W_{2} \wedge J+\bar{W}_{5} \wedge \Omega
\end{gathered}
$$

where the representations of the $W_{i}$ are as follows:

- $W_{1}$ is a complex zero-form in $1 \oplus 1$;

- $W_{2}$ is a complex primitive two-form, so it lies in $8 \oplus 8$;

- $W_{3}$ is a real primitive $(2,1) \oplus(1,2)$ form, so it lies in $6 \oplus \overline{6}$;

- $W_{4}$ is a real one-form in $3 \oplus \overline{3}$;

- $W_{5}$ is a complex $(1,0)$-form (notice that in (4.1) the $(0,1)$ part drops out), so its degrees of freedom are again $3 \oplus \overline{3}$.

These $W_{i}$ allow to classify quickly any $\mathrm{SU}(3)$ manifold. We will later define them in an alternative way using directly the spinor; that definition will be more natural for T-duality, but the $W$ 's are often better to analyze the type of the manifold. For example, notice that in (4.1) the exterior derivative $d$ does not satisfy the usual rule $d: \Omega^{p, q} \rightarrow \Omega^{p+1, q} \oplus \Omega^{p, q+1}$. For an almost complex manifold as we have here, there are also $(p+2, q-1)$ and $(p-1, q+2)$ contributions. Hence in (4.1) the $(3,0) \oplus(0,3)$ part of $d J$, namely $\operatorname{Im}\left(W_{1} \bar{\Omega}\right)$, and the $(2,2)$ part of $d \Omega$, which reads $W_{1} J^{2}+W_{2} \wedge J$. So we know actually that $W_{1}=W_{2}=0$ iff the manifold is complex. One can check indeed that the Nijenhuis tensor can be expressed in terms of $W_{1}$ and $W_{2}$. Other examples of the use of these $W$ 's abound in the literature. Notice also that the information of $d J$ and $d \Omega$ is a little redundant, as $W_{1}$ appears in both. 
Before we start computing, notice that from this classical definition it would be not obvious to guess transformation laws for $W$ 's, other than some qualitative features. There are two vectors, but the 8 and the 6 are different representations. If one thinks already at this stage about decomposing in base representations, guessing becomes easier, but one feels rapidly the need for a more solid ground. One way, which we pursue in this section, is to compute blindly. The other way is to put $J$ and $\Omega$ on a more symmetrical basis, using the formalism of Clifford(d, d), or, which is another manifestation of the same idea, to actually use the SU(3) invariant spinor directly. We do this in next section.

\subsection{Computations of torsions in the $T^{3}$ fibered case}

We can now compute $W$ 's from the expressions (2.12) and (2.13). This is done by doing contractions, partial or total, appropriate to isolate the component of interest. For example $W_{4}$ is computed contracting $J\lrcorner d J .^{3}$ First we give $W_{1}, W_{4}, W_{5}$, expressed in the holomorphic basis. ${ }^{4}$ Note that $W_{4}$ is real and $W_{5}$ holomorphic, so that $W_{4}=w_{i}^{4} d z^{i}+$ c. c. and $W_{5}=w_{i}^{5} d z^{i}$, while $W_{1}=w_{1}$ is a scalar. ${ }^{5}$ These components read:

$$
\begin{aligned}
w_{1} & =-\frac{i}{12} \epsilon^{i j k} V_{i \alpha}[d(V-i \lambda)]_{j k}^{\alpha} \\
w_{k}^{4} & =-\frac{1}{4} V^{\alpha j}\left[d V_{\alpha}\right]_{j k} \\
w_{k}^{5} & =-\frac{1}{4}\left\{V_{\alpha}^{j}[d(V+i \lambda)]_{j k}^{\alpha}-h^{\alpha \beta} \partial_{k} h_{\alpha \beta}\right\}
\end{aligned}
$$

where $[d(\cdot)]_{i j}=2 \partial_{[i}(\cdot)_{j]}$.

We now pass to $W_{2}$ and $W_{3}$. $W_{2}$ is a $(1,1)$-form, and $W_{3}$ is a real $(2,1) \oplus(1,2)$, and are written as

$$
W_{2}=w_{i j}^{2} d z^{i} \wedge d \bar{z}^{j}, \quad W_{3}=\frac{1}{2} w_{i j k}^{3} d z^{i} \wedge d \bar{z}^{j} \wedge d \bar{z}^{k}+\text { c. c. },
$$

However, since the representation 6 can be expressed not only as a primitive $(2,1)$ form, but also as a symmetric tensor with two holomorphic indices, we will give this latter expression for $W_{3}$. The way to pass from one to another is $w_{i j}^{3}=w_{i p q}^{3} \Omega^{p q}{ }_{j}$. This is already a little in the spirit of the different basis for intrinsic torsion that we will give later. Furthermore, these two matrices with indices $i j$ can actually be further decomposed in representation theory of the $S O(3)$ of the base. $w_{i j}^{2}$ has a symmetric and an antisymmetric part; the symmetric part does not drop out, it only contributes to $d y \wedge e$ part; the antisymmetric part can be dualized to a three dimensional vector $w_{2}^{i}=\frac{1}{2} \epsilon^{i j k} w_{j k}^{2}$. As for $W_{3}, w_{i j}^{3}=w_{\{i j\}^{0}}^{3}+\frac{1}{3} w_{t}^{3} g_{i j}$ is already symmetric but has a trace part $w_{t}^{3}$ on the three-dimensional base (of course, it is traceless in six dimensions).

$$
\begin{aligned}
w_{\{i j\}}^{2} & =\frac{1}{24} \epsilon^{p q k}[d(V-i \lambda)]_{p q}^{\alpha}\left[2 V_{k \alpha} g_{i j}-3 V_{j \alpha} g_{i k}-3 V_{i \alpha} g_{j k}\right] \\
w_{k}^{2} & =-\frac{1}{4} V_{\alpha}^{j}[d(V-i \lambda)]_{j k}^{\alpha}
\end{aligned}
$$

\footnotetext{
${ }^{3}$ Complete expressions for all five components of the intrinsic torsion for a metric of the form 2.1 can be found in appendix $\mathrm{A}$

${ }^{4}$ In what follows, we will denote $W$ 's in complex coordinates as lower case $w^{\prime}$ s. For example, $\bar{w}_{i j}^{2}$ is $\bar{W}_{m \bar{n}}^{2}$, even if we did not explicitly mark $i$ and $j$ as holomorphic and antiholomorphic indices in this expression. This is also true for the other components.

${ }^{5}$ As already emphasized, one has to bear in mind that the almost complex structure is in general not integrable, so that $d z^{i}$ is not to be understood as the differential of a hypothetical coordinate $z^{i}$.
} 


$$
\begin{aligned}
w_{\{i j\}^{0}}^{3} & =\frac{1}{24} \epsilon^{p q k}\left[d V_{\alpha}\right]_{p q}\left[2 V_{k}^{\alpha} g_{i j}-3 V_{j}^{\alpha} g_{i k}-3 V_{i}^{\alpha} g_{j k}\right] \\
w_{t}^{3} & =\frac{1}{8} \epsilon^{p q k}[d(V-3 i \lambda)]_{p q}^{\alpha} V_{k \alpha}
\end{aligned}
$$

Before turning to the T-duality transformations of components of the intrinsic torsion and the flux, we observe that the conditions for a supersymmetric vacuum with $H$ only (1.3) are not compatible in a nontrivial way with the expressions above, as we anticipated in the introduction. For example, demanding $W_{1}=W_{2}=0$ sets $\lambda$ and $V$ to constants.

\subsection{T-duality}

It is now easy to see what the transformation rules of the $W$ 's are. Decomposing in base representations says essentially where to look. One sees immediately that the various three-dimensional vectors and symmetric matrices are all similar. Before spelling this out, one should however stress that the full six-dimensional quantities have a more complicated transformation rule. As explained in section 3.2 due to presence of $\lambda$ in $e^{\gamma}, d z^{i}=d y^{i}-i V_{\gamma}^{i} e^{\gamma}$ on the original manifold does not map exactly to $d z$ on the mirror side.

With this important caveat in mind, let us proceed to give T-duality transformations. As we said, many of the expressions we have for $W$ 's are similar (see appendix $\mathrm{A}$. The differences are mainly because of $V_{\alpha}$ versus $V^{\alpha}$. This is already good, as these quantities are exchanged by $\mathrm{T}$ duality (2.9). One also sees that some of the quantities contain $\lambda$, that after T-duality become $B$ as we just recalled. So, we are led naturally to complexify some of the torsions adding $d B$ projected in the appropriate representation. As this projections are verbatim those we did for $d J$ in previous subsection, this step is trivial. Thus defining components for $H$ as for other forms ${ }^{6}$

$$
H=-\frac{3}{2} \operatorname{Im}\left(H_{1} \bar{\Omega}\right)+H_{4} \wedge J+H_{3}
$$

we find the transformations:

$$
\begin{gathered}
w_{1}-i h_{1} \longleftrightarrow-\overline{\left(w_{1}-i h_{1}\right)} \\
w_{\{i j\}}^{2} \longleftrightarrow\left(w_{3}+i h_{3}\right)_{\{i j\}^{0}}, \\
w_{k}^{5}-\frac{1}{4} h^{\alpha \beta} \partial_{k} h_{\alpha \beta}=\bar{w}_{k}^{2} \longleftrightarrow\left(w_{4}-i h_{4}\right)_{k} .
\end{gathered}
$$

describing the mixing of the components of the flux and of the intrinsic torsion under mirror symmetry.

The central role in the mirror/T-duality transformation (4.11) is obviously played by $W_{2}$ (a component of the torsion associated with the non-integrability of the complex structure). It splits in two different pieces upon restriction to the base and the respective mixing of the two parts of $W_{2}$ with complexified $H_{3}$ and $H_{4}$ is an essential ingredient of the mirror map.

We will now try to rederive and generalize to generic geometries these results from a different point of view, using spinors rather than differential forms.

\section{Spinorial basis}

The idea is that the same information we have in $d J$ and $d \Omega$ are contained in $D_{M} \epsilon$. Doing the effort of reexpressing torsions in these terms pays off for several reasons. First of all, the combinations

\footnotetext{
${ }^{6}$ The explicit expressions for components of $H$ in the $T^{3}$-fibered geometry can be found in appendix $\mathrm{A}$ We labeled these components so that they match the corresponding ones in $d J . H_{1}$ is then the $1 \oplus 1$ complex scalar, $H_{3}$ the $6 \oplus \overline{6}$ real 3 -form and $H_{4}$ the $3 \oplus \overline{3}$ real 1 -form.
} 
that appear in $D_{M} \epsilon$ transform better. Second, they might be useful in future occasions to analyze the geometry behind a given supersymmetry transformation without even having to bother to construct bilinears. In particular, we can find from this approach immediately the conditions (1.3) for supersymmetric vacua with $H$.

One proceeds in the following way. What we call $\epsilon$ in what follows is the $\mathrm{SU}(3)$ invariant spinor, which can be furthermore decomposed by chirality as $\epsilon_{+}+\epsilon_{-}$. Again, if we were on a manifold of $\mathrm{SU}(3)$ holonomy, we would have a covariantly constant spinor, $D_{M} \epsilon=0$. This is not the case, but still decomposing $D_{M} \epsilon$ into representations will give us a measure of how far we are from $\mathrm{SU}(3)$ holonomy. The way of decomposing $D_{M} \epsilon$ into representations is again implicit in the literature. On a $\mathrm{SU}(3)$ invariant manifold, a basis for spinors is given by $\epsilon_{ \pm}$and $\gamma_{M} \epsilon_{ \pm}$(or alternatively we can trade $\epsilon_{ \pm}$with $\epsilon$ and $\gamma \epsilon$ ). So, for example, anything else in Clifford algebra acting on $\epsilon$, say $\gamma^{M_{1} \ldots M_{n}}$, can be reexpressed in terms of this basis. Explicit formulas for this are known (see for example [6]; in [14] a complete set of these equations are provided, along with the simple group theoretical description of how to get them, for the case of seven-manifolds with $\mathrm{G}_{2}$ structure). We will not however need them here, it is enough to know that this decomposition can be done. Actually, with one exception: the relation $\gamma^{M} \gamma \epsilon=i J^{M}{ }_{N} \gamma^{N} \epsilon$ can be used to eliminate one possible term. So we can write in general

$$
D_{M} \epsilon=\left(q_{M}+i \tilde{q}_{M} \gamma+i q_{M N} \gamma^{N}\right) \epsilon .
$$

The real $q$ 's that we have defined in this equation are just another definition of intrinsic torsion. To see that they can be compared with the $W$ 's above, it suffices to use group theory. $q_{M}$ and $\tilde{q}_{M}$ are vectors, $3 \oplus \overline{3}$; as to $q_{M N}$, it can be decomposed into $(3 \oplus \overline{3})^{\otimes 2}=(6 \oplus \overline{3}) \oplus(\overline{6} \oplus 3) \oplus(8 \oplus 1) \oplus(8 \oplus 1)$. We see that all the representations of the $W$ 's are present. There is one redundancy, since we get three vectors $\left(q_{M}, \tilde{q}_{M}\right.$ and one from $\left.q_{M N}\right)$. The objects we get in this way are the same as the $W$ 's up to factors. Qualitatively we could stop here; in the present context we are actually interested in getting the factors, as they are important for being able to express $q$ 's in terms of $W$ 's explicitly. This is done as follows. After having decomposed $q_{M N}$ as above, we can define $J$ and $\Omega$ as bilinears as

$$
\epsilon^{\dagger} \gamma_{M N} \gamma \epsilon=i J_{M N}, \quad-i \epsilon^{\dagger} \gamma_{M N P}(1+\gamma) \epsilon=\Omega_{M N P} .
$$

One can now compute their exterior derivative using (5.1). Comparing the result with (4.1) gives the desired coefficients. The result is

$$
\begin{aligned}
q_{M N}= & \frac{1}{4}\left(W_{1}^{+} G_{M N}+W_{1}^{-} J_{M N}\right)+\frac{1}{8}\left(\Omega_{M N P}{\overline{\left(W_{5}-2 P W_{4}\right)}}^{P}+\text { c. с. }\right) \\
& \quad+\frac{1}{4}\left(-J_{M}^{P} W_{P N}^{2,+}+W_{M N}^{2,-}\right)+\frac{1}{8} \operatorname{Im}\left(W_{M N}^{3}\right) \\
= & \operatorname{Re}\left[\frac{1}{2} W_{1} \bar{P}_{M N}+\frac{1}{4} \Omega_{M N P}\left(\bar{W}_{5}-2 W_{4}\right)^{P}+\frac{i}{2} \bar{P}_{M}{ }^{P} W_{P N}^{2}-\frac{i}{8} W_{M N}^{3}\right] \\
q_{M}+i \tilde{q}_{M}= & {\left[\bar{W}_{5}-\bar{P} W_{4}\right]_{M} }
\end{aligned}
$$

where $W_{i}=W_{i}^{+}-i W_{i}^{-}$as usual in the literature, and we have defined $W_{M N}^{3}=W_{M P Q}^{3} \Omega^{P Q}{ }_{N}$ and used a holomorphic projector $P=\frac{1}{2}(1-i J)$. We had observed in the previous section that the split of $W_{2}$ in two parts upon restriction to the base is crucial in the mirror transformation. Here we can see that the split nature of $W_{2}$ reveals itself in covariant six-dimensional expressions: $W_{2}^{+}$ and $W_{2}^{-}$enter respectively into the symmetric and antisymmetric parts of $q_{M N}$.

It is worth recording the same expression in holomorphic/antiholomorphic basis:

$$
q_{m n}=-\frac{i}{16} w_{m n}^{3}+\frac{1}{8} \Omega_{m n p}\left(w_{5}-2 w_{4}\right)^{p}, \quad q_{m \bar{n}}=-\frac{i}{4} \bar{w}_{m \bar{n}}^{2}+\frac{1}{4} \bar{w}_{1} g_{m \bar{n}} .
$$

And for the remaining vector:

$$
q_{m}-i \tilde{q}_{m}=\left(w_{5}-w_{4}\right)_{m} .
$$


The quantities we have defined so far would not be expected to behave nicely under T-duality, for the following simple reason. The transformation laws we have computed in (4.11) have, as one would expect also from the arguments in [1] and from (1.1), the feature of exchanging some torsions with $H$. Therefore we have to add a dependence on $H$ to the covariant derivative in (5.1). Then also the $q$ defined in (5.1) will change and (5.1) will become

$$
D_{M}^{H} \epsilon=\left(Q_{M}+i \tilde{Q}_{M} \gamma+i Q_{M N} \gamma^{N}\right) \epsilon
$$

We have defined $D^{H}$ (and as a consequence the $Q$ 's) in such a way as to find good T-duality transformation properties afterwards. Not too surprisingly, we have found that the best definition is exactly the same as the one which appears in supergravity supersymmetry transformations: $D_{M}^{H} \equiv\left(D_{M}+\frac{1}{8} H_{M N P} \gamma^{N P}\right)$. We find then

$$
\begin{aligned}
Q_{M N}=\operatorname{Re}[ & \frac{1}{2}\left(W_{1}+3 i H_{1}\right) \bar{P}_{M N}+\frac{1}{4} \Omega_{M N P}\left(\bar{W}_{5}-2\left(W_{4}+i H_{4}\right)\right)^{P} \\
& \left.+\frac{i}{2} \bar{P}_{M}^{P} W_{P N}^{2}-\frac{i}{8}\left(W^{3}+i H^{3}\right)_{M N}\right] .
\end{aligned}
$$

So, adding $H$ as $D \rightarrow D^{H}$ complexifies $W$ as $W+i H$, though at the end the Re in (5.8) makes the $Q$ 's real. It should also be possible to write directly a formula for the (con)torsion, as an alternative to formulas for the $q$ 's that we have given. The fact that $H$ appears as $H_{M N P} \gamma^{N P}$ tells us already that this formula will have a piece $K_{M N P}=d J_{M N P}+\ldots$ that will combine with $H$. As we will not need it here, we do not pursue this. Notice also that the $G_{2}$ analogue of what we just did for $H$ is discussed in detail in [14 for the $G_{2}$ case.

The fact that the natural combination for T-duality and for supersymmetry is the same will be useful later, when we will try to extend our results to the general case. Then this is also a good place to see that of course the conditions for supersymmetry in the case with $H$ only (1.3) can be recovered from the spinor equation. To have supersymmetry it is enough that one chirality, say $\epsilon_{+}$, is annihilated by $D^{H}$. We have the expressions

$$
\begin{array}{ll}
D_{m}^{H} \epsilon_{+}=\left(Q_{m}+i \tilde{Q}_{m}\right) \epsilon_{+}+i Q_{m n} \gamma^{n} \epsilon_{-} & D_{m}^{H} \epsilon_{-}=\left(Q_{m}-i \tilde{Q}_{m}\right) \epsilon_{-}-i Q_{m \bar{n}} \gamma^{\bar{n}} \epsilon_{+} \\
D_{\bar{m}}^{H} \epsilon_{+}=\left(Q_{\bar{m}}+i \tilde{Q}_{\bar{m}}\right) \epsilon_{+}+i Q_{\bar{m} n} \gamma^{n} \epsilon_{-} & D_{\bar{m}}^{H} \epsilon_{-}=\left(Q_{\bar{m}}-i \tilde{Q}_{\bar{m}}\right) \epsilon_{-}-i Q_{\bar{m} \bar{n}} \gamma^{\bar{n}} \epsilon_{-}
\end{array}
$$

Notice that $Q_{m \bar{n}}$ and $Q_{\bar{m} \bar{n}}$ have disappeared from $D^{H} \epsilon_{+}$, because $\epsilon_{-}$, being a Clifford vacuum, is annihilated by $\gamma^{\bar{n}}$. From this one obtains directly that the complexified $Q_{m n}$ and $Q_{\bar{m} n}$ have to vanish. These will say that the complexified $W_{3}$ has to be purely antiholomorphic, which in more usual terms means of type $(1,2)$ (this is the condition $\left.W_{3}=* H_{3}\right)$ and that $W_{2}$ has to vanish. The vectors require a little more care because usually the dilaton is rescaled in the metric (as a warping) and in the spinor itself. More generally it is clear that one can use gamma matrices identities mentioned above to reduce the expression to a form like (5.1), and then use (5.4) or (5.5).

For us the main advantage of having computed these quantities is to compare with T-duality transformations given in previous sections, although we will see shortly how these supersymmetry considerations can play a role in understanding the general case (without $T^{3}$ fibration structure). We can restrict the free index in (5.1) to be on the base, $M=i$, and furthermore apply a chirality projector

$$
D_{i} \epsilon_{+}=\hat{Q}_{i} \epsilon_{+}+i \hat{Q}_{i j} \gamma^{j} \epsilon_{-}, \quad D_{i} \epsilon_{-}=\overline{\hat{Q}}_{i} \epsilon_{-}+i \overline{\hat{Q}}_{i j} \gamma^{j} \epsilon_{+} .
$$

having introduced hatted quantities for restrictions to the base. The quantities $\hat{Q}_{i}$ and $\hat{Q}_{i j}$ in these expressions turn out to transform neatly under T-duality:

$$
\hat{Q}_{i} \longrightarrow-\overline{\hat{Q}}_{i}, \quad \hat{Q}_{i j} \longrightarrow-\overline{\hat{Q}}_{i j},
$$


with the expressions

$$
\begin{aligned}
\hat{Q}_{i}= & Q_{i}+i \tilde{Q}_{i}=\left(\bar{W}_{5}-\frac{1}{2}\left(W_{4}-i H_{4}\right)\right)_{i} \\
\hat{Q}_{i j}= & Q_{i j}-i Q_{i \alpha} V_{j}^{\alpha}=2 \bar{P}_{j}^{M} Q_{i M} \\
= & \frac{1}{4}\left[\bar{W}_{1}+3 i \bar{H}_{(1)}+\frac{i}{12} \bar{P}^{i M}\left(W_{3}-i H_{3}\right)_{M i}\right] g_{i j}-\frac{i}{4}\left[\bar{W}_{\{i j\}}^{2}-\frac{1}{4} \bar{P}_{i}^{M}\left(W_{3}-i H_{3}\right)_{M j}\right] \\
& \quad+\frac{i}{2} \epsilon_{i j k}\left[W_{5}-W_{4}-i H_{4}-\frac{1}{2} \bar{W}_{2}\right]^{k}
\end{aligned}
$$

This means that at an effective level the rule tells us $\epsilon_{+} \leftrightarrow \epsilon_{-}$.

We should remark that working so far with a finite-size $T^{3}$-fiber, we have extra (nowhere vanishing) vector fields, and thus reduces structure. This may in particular allow to locally preserve supersymmetry even when conditions (1.3) are violated. Since when fibers degenerate this restricted stricture no longer exists, we avoided making explicit use of it, even though doing restrictions to the base manifolds implicitly uses the existence of a restricted structure. It is reasonable to expect that the results based on representations are valid over the entire moduli space, and thus next we turn to the six-dimensional covariantization of mirror transformation (4.11).

\subsection{Approaches to the general case}

At this point it is natural to wonder if we have enough information to simply guess what mirror symmetry should be in the general case. We have a precise set of transformation rules in the case of $T^{3}$ fibrations, and we also know that supersymmetric vacua should be sent to supersymmetric vacua. As we remarked above, T-duality is induced by an exchange of $\epsilon_{+}$with $\epsilon_{-}$. Since we also have $\gamma^{\bar{m}} \epsilon_{-}=0$, these two facts together would suggest following proposal naturally generalizing (5.11):

$$
Q_{m n} \longleftrightarrow-Q_{m \bar{n}}, \quad Q_{m} \longleftrightarrow-\bar{Q}_{m}
$$

We noticed above that representations of $W$ 's do not match in such a way as to suggest immediately a transformation law. In the $\mathrm{T}$-duality approach above this was solved by decomposing further in representations of the $\mathrm{SO}(3)$ of the base. The proposal (5.14), on the contrary, gets around this problem collecting together $\mathrm{SU}(3)$ representations rather than decomposing them further: qualitatively, $6 \oplus \overline{3} \leftrightarrow 8 \oplus 1$.

Let us now check that this proposal for mirror symmetry agrees with T-duality and with supersymmetry, as we just required. First of all, (5.14) agrees with the exchange (5.11). Indeed we have

$$
\hat{Q}_{M \bar{n}}=P_{m}{ }^{P} \hat{Q}_{P \bar{n}}+\bar{P}_{\bar{m}}^{P} \hat{Q}_{P \bar{n}}=2 Q_{m \bar{n}}+2 Q_{\bar{m} \bar{n}} ;
$$

similarly one can consider the transformation of $Q_{m}=\hat{Q}_{m}$.

Turning now to supersymmetry, the two transformations in (5.14) induce simply

$$
D_{m}^{H} \epsilon_{+} \longrightarrow-D_{m}^{H} \epsilon_{-} .
$$

So if only $H$ is present we are sending $D^{H} \epsilon_{+}=0$ to $-D^{H} \epsilon_{-}=0$; in the latter case supersymmetry is of course still preserved. In this form the duality might seem a little tautological, in the sense that it sends a supersymmetric vacuum in another one in an obvious way. Compare however with the usual mirror symmetry: a Calabi-Yau is sent to another Calabi-Yau, and the nontriviality lies in the exchange of Kähler and complex structure moduli. This should be happening for vacua with $H$ only as well, and in a sense this would be yet another check to do; we will comment on this in next section.

Coming back to checking compatibility with supersymmetry, the situation becomes more complicated with RR fluxes, because the latter also transform, and one would have to check that they 
do it in a way compatible with the one we are giving for geometry and $H$. This can be elaborated as follows. Just as the entire NS contribution to the covariant derivative of the invariant spinor got summarized in $Q$ 's (see (5.8)), the RR contribution can be accounted by introduction of similar objects, $R_{M}, \tilde{R}_{M}$ and $R_{M N}$ with a group decomposition matching that of $Q$ 's. On supersymmetric backgrounds, the total action of the covariant derivative of the invariant spinor should be zero and thus $R=-Q$. Thus from this point of view the mirror transformation of the RR sector can also be brought to the form (5.14). From other side, in the $T^{3}$ fibered case, one could use the known transformation rules of RR fields. From the above, it is clear that the natural way to do this check in general would be to consider RR fields not as sums of forms but as bispinors, expressing for example in terms of the latter also supersymmetry transformations.

Even after all these motivations, the proposal (5.14) stands as a conjecture, and there would be other possible checks to be made. One possibility is to use again the formalism of Clifford $(6,6)$ spinors. One can give an alternative definition of torsions, that we have not mentioned so far, using the Clifford $(6,6)$ spinors $e^{i J}$ and $\Omega$. Schematically one gets

$$
D_{M} e^{i J}=q_{M} e^{i J}+\operatorname{Im}\left(q_{M}^{(2)} \cdot \Omega\right), \quad D_{M} \Omega=\left(q_{M}+i \tilde{q}_{M}\right) \Omega+q_{M}^{(2)} \cdot e^{i J} .
$$

In these equations, $q_{M}^{(2)}$. is the Clifford product of $q_{M N}$ using only second index. These formulas seem indeed to be consistent with the general rule (5.14) given above.

\section{Applications and examples}

In this section we analyze some simple consequences of the mirror symmetry transformation that we have proposed. Apart from the case in which only geometry and $B$-field are present, the situation will be different from the usual one for Calabi-Yau's in that RR fluxes will transform, and so solutions with some types of fluxes switched on get mapped generically to solutions with other types of fluxes. On top of this we should also have the usual exchange of Kähler and complex structure moduli, in the sense of (1.1). Simple checks of both claims have been listed in previous section; here we take these statements for granted and examine the consequences.

The natural starting point is to check how the picture developed so far reduces to known cases. We start from a brief discussion of an example which has already been mentioned, and involves a CY manifold with $B$-field turned on. This case was considered in 1 in great detail. Since the intrinsic torsion vanishes on CY, we start from $Q_{M N}$ built purely from components of $H$. The $Q_{m \bar{n}}$ gets a single contribution from $H_{1}$. If we follow [1] and look for a purely geometrical mirror, on the mirror side we may have non-zero $\tilde{W}_{3}$ and $\tilde{W}_{4}-\tilde{W}_{5}=0$. Looking at $Q_{m n}$, we see that the reality of remaining components of the flux ensures that on the mirror side only $\tilde{W}_{1}^{-}$and $\tilde{W}_{2}^{-}$survive. This agrees with [1] up to a conventional \pm exchange. So we recover as a particular case the half-flat geometries and the $\mathrm{G}_{2}$ lifts discussed in [16, 17. Note that neither the starting configuration, nor its mirror are vacua but rather domain walls.

The simplest background is when the $B$-field is turned off and we just deal with Calabi-Yau geometry. This case was also discussed in section 3 where we recover the exchange of the complex structure and (the exponentiated) Kähler form for mirror Calabi-Yau manifolds. An exchange of complex and Kähler moduli for a metric of the form (2.1) with $\lambda=0$ and the integrability properties of its complex structure were studied in [15. Here we easily see that the exchange of the $e^{i J}$ and $\Omega$ is accompanied by an exchange of their integrability conditions.

Without turning RR fields on, we can also consider yet another possibility of vacua. ${ }^{7}$ These cases are to obey the conditions given in (1.3). In our language these conditions read $Q_{m n}=0=$ $Q_{\bar{m} n}$. What one gets by the proposal (5.14) is the condition $Q_{m \bar{n}}=0=Q_{\bar{m} \bar{n}}$, which is obviously

\footnotetext{
${ }^{7}$ Here and in the parts with also RR on, the word vacuum should be understood with the usual grain of salt: no-go theorems force us to consider noncompact or singular cases, or to hope (in a less well-defined way) that some of the features analyzed here will survive after taking into account higher-derivatives corrections.
} 
isomorphic to it (see the comments in previous section). Less trivial is the statement that complex and Kähler moduli are exchanged. To check this one would have first of course to know by what groups moduli spaces are computed.

This check we will not be able to perform here, and we limit ourselves to some comments. First of all, in general moduli spaces of solutions with fluxes are likely not to be simply factorized in Kähler and complex part. This is because, unlike the Calabi-Yau case, the conditions are no longer $d J=0=d \Omega$, but something involving torsions; and the definition of torsions 4.1] mixes $\Omega$ and $J$. Also, in the Calabi-Yau case the fact that the conditions were of simple closure allowed to reduce the counting to a cohomology problem. In general, here, we are dealing with conditions involving projections $P_{\text {rep }} d J$ and $P_{\text {rep }} d \Omega$, where $P_{\text {rep }}$ is a projector on a certain representation. These conditions mean roughly that a form is closed "up to" a contribution from the other form, schematically $d J=$ operator $(\Omega)$. In general it should be possible to restate this as the cohomology of a double complex. Coming back to the case with $H$ only switched on, a preliminary analysis of moduli spaces was sketched in [18, following ideas in [19. Indeed the $H$-twisted cohomology groups proposed there are total cohomologies of a double complex with $\bar{\partial}$ and $H^{2,1} \wedge$ as differentials. We will unfortunately not say more on this here, but plan to come back on the issue in the future. For now we just observe that, in known examples, fluxes fix complex structure moduli. These considerations tell us that in a mirror picture Kähler moduli will be fixed.

Type B solution for IIB strings presented in 20 provides with another related case flux compactifications with the back-reaction taken into account. The metric now is conformally CY, and RR-fluxes are turned on as well. In addition, supersymmetry conservation imposes restrictions on $H$-flux, which now turns out to be primitive. We will not attempt here to present a complete analysis of the mirror transformation and will ignore the RR sector (which mirror symmetry maps to RR fields in Type IIA theory). Thus our starting data include $W_{4} \sim W_{5}$ and $H_{4}$. Note that this means in particular that we have $Q_{m \bar{n}}=0$, and thus we need $\tilde{Q}_{m n}=0$ on the mirror side. The two previous examples have this feature: we could either take $\tilde{H}=0$ and $\tilde{W}_{3}=\tilde{W}_{4}-\tilde{W}_{5}=0$ or have $\tilde{H}$ with imaginary selfdual primitive part and geometry given by $\tilde{W}_{3}=* \tilde{H}_{3}$ and $2 \tilde{W}_{4}=\tilde{W}_{5}=2 d \tilde{\phi}=2 i \tilde{H}_{4}$ as in 8 . However differently from previous cases $Q_{m n} \neq 0$ and this results in an additional nonintegrability of the complex structure on the mirror side (in particular, $\tilde{W}_{2}^{-}$cannot be zero now). Of course, explicit constructions of such IIA string backgrounds would be of some interest.

The last application we will discuss here concerns the possibility of lifting the SU(3) mirror symmetry picture to the $\mathrm{G}_{2}$-structure case. We could start from IIA string theory in a monopole background and lift it to M-theory, using the explicit relations between the components of intrinsic torsion for $\mathrm{SU}(3)$ and $\mathrm{G}_{2}$ for $\mathrm{U}(1)$-fibered manifolds. The components of the torsion for the representations 1,7 and 27 get complexified by the corresponding representations of the $G_{4}$-flux. The analogy with the $\mathrm{SU}(3)$ case is rather close. There as well there was a number of components of the intrinsic torsion that get complexified by the $H$-flux; mirror symmetry then mixed these with the components corresponding to representations that are not contained in the flux (essentially $8 \oplus 8$ in that case, with some extra subtleties having to do with $3 \oplus \overline{3}$ appearing twice). In the $\mathrm{G}_{2}$ geometry, 14 is such a representation, and the corresponding component of the torsion is the lifting of $W_{2}^{-}$17, 21, the component of SU(3)-torsion central in the exchange with the NS flux. Once more one would be hoping that going to spinorial basis and writing for the invariant spinor the twisted covariant derivative will lead to a covariant expression for a mirror transformation for the $\mathrm{G}_{2}$ geometry. Indeed, as in (5.1) the torsion for the $\mathrm{G}_{2}$-structure manifolds is also encoded in a covariant derivative $D_{M} \epsilon=\left(q_{M}+i q_{M N} \gamma^{N}\right) \epsilon$, where $q$ 's are real. Then the eleven-dimensional supersymmetry transformations restricted to seven-dimensions twist the covariant derivative by a term $\frac{i}{3} G_{M} \epsilon-\frac{1}{3}\left(2 G \delta_{M N}+G_{M N}+2 G_{N M}\right) \gamma^{N} \epsilon$, where we have defined $G \equiv \frac{1}{4 !} G_{M N P Q}(* \Phi)^{M N P Q}$, $G_{M} \equiv \frac{1}{3 !} G_{M N P Q} \Phi^{N P Q}, G_{M N} \equiv \frac{1}{3 !} G_{M P Q R}(* \Phi)^{P Q R}{ }_{N}$ using the associative form $\Phi$. Putting all together we arrive at the twisted operator

$$
D_{M}^{G} \epsilon=\left(Q_{M}+i Q_{M N} \gamma^{N}\right) \epsilon
$$

which we can now use to extend the $\mathrm{SU}(3)$ mirror symmetry proposal. Indeed, the $\mathrm{G}_{2}$ analogue of 
(5.14) can be written as

$$
Q_{[M N]}^{+} \longleftrightarrow-Q_{[M N]}^{-} \quad Q_{\{M N\}} \longrightarrow-Q_{\{M N\}}
$$

where \pm denote selfdual and antiselfdual representations respectively. Note that only the former is complexified by $G$-flux, and (6.1) exchanges 14 with $7+7$. In view of this, we may go back to (5.14) and note that there as well, modulo the trace part, mirror symmetry can be thought of as an exchange of selfdual and antiselfdual matrices (à la Hermitian Yang-Mills).

\section{Discussion}

We conclude by mentioning some open technical and conceptual problems. Throughout the paper we have worked with a $B_{\alpha \beta}=0$ case. Obviously, this choice simplifies greatly the T-duality transformation. The reason for this is most clear on the spinorial picture. As shown in [12, the only change in the simple $\mathrm{T}$-duality transformations used above (see section 3.2 ) occurs when $B_{\alpha \beta}$ component of the $B$-field is nonzero. In this case we have to use instead

$$
\psi_{+} \rightarrow \psi_{+}, \quad \psi_{-} \rightarrow e^{E} \gamma_{f} \psi_{-}
$$

where $E^{\alpha \beta}$ is defined in (2.5). We have here a gamma matrix exponential of $E \equiv \frac{1}{2} E^{\alpha \beta} \gamma_{\alpha \beta}$ which has the same form of the kappa-symmetry $\Gamma$ operator; in the power series expansion the products of all gamma matrices are antisymmetrized.

Note that without a $B_{\alpha \beta}$ component, there is a certain ambiguity in the choice of T-duality invariants (5.12). The ambiguity is in the of complexification by $H$ in $Q$ 's. He have chosen everywhere the plus sign (and correspondingly $\mathrm{T}$-dual expressions which become complex conjugates) for the following reason. The singlet representation allows a simple calculation even with a non-vanishing $B_{\alpha \beta}$. The result is then the first formula in (4.11), which fixes the ambiguity. For all other components we have chosen the complexification rule consistent with that of $W_{1}$, hence the choice of sign in the definition of the twisted covariant derivative (5.7). The $\mathrm{T}$-duality rule for the spinors given above should allow to lift restrictions from the $B$-field and verify this explicitly. We would like to emphasize though that this restriction is of technical nature - for a number of applications the $B$-field is generic enough. First, the $H$-flux contains all the representations it can. Second, in the holomorphic coordinate basis it is not hard to see that $B$ is of generic type and contains both $(1,1)$ and $(2,0)$ components. The latter is important for several aspects of topological B-branes (see [22] for a recent discussion, in which also Clifford(d,d) spinors appear) and mirror symmetry [23.

Clearly there are two directions in which our results have to be extended. As mentioned many times we have worked with a $T^{3}$ fibration with finite-size fibers (and thus had a luxury of having extra vector fields without zeros) and most of our formulae explicitly involve restrictions to the base of the fibration. At the end we succeeded in finding a basis in which the mirror/T-duality transformations can be covariantized and written over the entire six-dimensional manifold. The final simple rule for the mirror transformation

$$
Q_{m n} \longleftrightarrow-Q_{m \bar{n}}, \quad Q_{m} \longleftrightarrow-\bar{Q}_{m}
$$

is of group-theoretical nature, and we conjectured it to be true for general geometries, even without fibration structure at all, not even locally. In particular it should also work when there is a fibration but with singular fibers. From other side, singular $T^{3}$ fibers hold the key to SYZ picture, and would be extremely important to understand their fate in any generalization of SYZ.

Finally, one would like to complete the picture by incorporating D-branes. A better understanding of submanifolds in generalized CY manifolds as well as vector bundles on these would be essential preliminaries. Extending the picture developed in [5] for the exchange of branes (a pair of calibration and bundle conditions) and T-duality to generalized CY case would be of great interest. We may recall once more that in SYZ picture both mirror manifolds appear as moduli 
spaces for D-branes wrapping (sub)manifolds. One may hope that eventually developing the picture of D-brane moduli spaces in geometries with NS fluxes may lead to refining the proposal for mirror symmetry presented here.

Acknowledgments. We would like to thank Peter Kaste for participation in early stages of this work, and Mariana Graña and Fawad Hassan for useful conversations. This work is supported in part by EU contract HPRN-CT-2000-00122 and by INTAS contracts 55-1-590 and 00-0334.

\section{A Intrinsic torsion for $T^{3}$-fibered manifolds}

The components of the intrinsic torsion are defined by

$$
\begin{aligned}
d J & =-\frac{3}{2} \operatorname{Im}\left(W_{1} \bar{\Omega}\right)+W_{4} \wedge J+W_{3}, \\
d \Omega & =W_{1} J^{2}+W_{2} \wedge J+\bar{W}_{5} \wedge \Omega .
\end{aligned}
$$

They can be computed using contractions (\lrcorner$)$ with $J$ and $\Omega$ :

$$
\begin{aligned}
& \left.\left.\left.W_{1}=\frac{4}{3} J^{2}\right\lrcorner d \Omega=-\frac{4 i}{3} \Omega\right\lrcorner d J=\frac{1}{3} \epsilon_{A B C}\left(E^{A} \wedge E^{B}\right)\right\lrcorner d E^{C} \\
& =-\frac{i}{12} \epsilon^{i j k} V_{i \alpha}[d(V-i \lambda)]_{j k}^{\alpha} \\
& \left.W_{2}=4 J\right\lrcorner\left[d \Omega-W_{1} J^{2}-\bar{W}_{5} \wedge \Omega\right] \\
& =\frac{1}{12} \epsilon^{i j k}[d(V-i \lambda)]_{j k}^{\alpha}\left[g_{p q} V_{i \alpha}-3 g_{p i} V_{q \alpha}\right] d z^{p} \wedge d \bar{z}^{q} \\
& W_{3}=d J+\frac{3}{2} \operatorname{Im}\left(W_{1} \bar{\Omega}\right)-W_{4} \wedge J \\
& =\frac{3}{8} V_{i \alpha}\left[d \lambda^{\alpha}\right]_{j k} d y^{i} \wedge d y^{j} \wedge d y^{k} \\
& -\frac{1}{4}\left[d V_{\alpha}\right]_{i k}\left[\frac{3}{2} \delta_{j}^{k} \delta_{\beta}^{\alpha}+V_{j}^{\alpha} V_{\beta}^{k}-2 V^{k \alpha} V_{j \beta}\right] d y^{i} \wedge d y^{j} \wedge e^{\beta} \\
& +\frac{1}{4}\left[d \lambda^{\alpha}\right]_{j k}\left[\frac{1}{2} V_{i \alpha} V_{\beta}^{j} V_{\gamma}^{k}-\delta_{i}^{j} h_{\alpha \beta} V_{\gamma}^{k}\right] d y^{i} \wedge e^{\beta} \wedge e^{\gamma} \\
& -\frac{1}{8} V_{\beta}^{i} V_{\gamma}^{j}\left[d V_{\alpha}\right]_{i j} e^{\alpha} \wedge e^{\beta} \wedge e^{\gamma} \\
& =\frac{1}{16}\left\{-i\left[d V_{\alpha}\right]_{j k} V_{i}^{\alpha}+i\left[d V_{\alpha}\right]_{i j} V_{k}^{\alpha}+i\left[d V_{\alpha}\right]_{k i} V_{j}^{\alpha}+i\left[d V_{\alpha}\right]_{j l} V^{l \alpha} g_{i k}-i\left[d V_{\alpha}\right]_{k l} V^{l \alpha} g_{i j}\right. \\
& \left.+i\left[d \lambda^{\alpha}\right]_{j k} V_{i \alpha}+i\left[d \lambda^{\alpha}\right]_{i j} V_{k \alpha}+i\left[d \lambda^{\alpha}\right]_{k i} V_{j \alpha}\right\} d z^{i} \wedge d \bar{z}^{j} \wedge d \bar{z}^{k}+\text { c. c. } \\
& \left.W_{4}=2 J\right\lrcorner d J=\frac{1}{2} V^{\alpha k}\left[d V_{\alpha}\right]_{j k} d y^{j} \\
& =\frac{1}{2} h^{\alpha \beta}\left[d h_{\alpha \beta}-\mathcal{L}_{g V_{\alpha}} V_{\beta}\right]
\end{aligned}
$$




$$
\begin{aligned}
W_{5}^{(1,0)} & =-\Omega\lrcorner d \bar{\Omega} \\
& =\frac{1}{4}\left\{V_{\alpha}^{k}[d(V+i \lambda)]_{j k}^{\alpha}+h^{\alpha \beta} \partial_{j} h_{\alpha \beta}\right\} d z^{j} \\
& =\frac{1}{4}\left\{\left[h_{\alpha \beta} \mathcal{L}_{g V^{\alpha}} V^{\beta}\right]_{j}-i V_{\alpha}^{k}[d \lambda]_{j k}^{\alpha}\right\} d z^{j}
\end{aligned}
$$

where $d z^{j}=d y^{j}-i V_{\gamma}^{j} e^{\gamma}$.

In the last two expressions, we have used the Lie derivative $\mathcal{L}$, which is defined by

$$
\mathcal{L}_{X} Y=[X, Y]=\left[X^{i} \partial_{i} Y^{j}-Y^{i} \partial_{i} X^{j}\right] \partial_{j}, \quad \mathcal{L}_{X} \omega=\left[X^{i} \partial_{i} \omega_{j}+\omega_{i} \partial_{j} X^{i}\right] d y^{j},
$$

on the vector field $Y$ and the 1-form $\omega$, with respect to the vector field $X$. We wrote $V^{\beta}$ and $V_{\beta}$ for the 1-forms $V_{j}^{\beta} d y^{j}$ and $V_{j \beta} d y^{j}$, while $g V^{\alpha}$ and $g V_{\alpha}$ are the vector fields $V^{i \alpha} \partial_{i}$ and $V_{\alpha}^{i} \partial_{i}$.

We also give here the components of the $H$ field

$$
\begin{aligned}
H= & d B_{2} \\
= & \frac{1}{2} \partial_{k} B_{\alpha \beta} d y^{k} \wedge e^{\alpha} \wedge e^{\beta}+\left[\partial_{k} B_{i \alpha}-B_{\alpha \beta} \partial_{k} \lambda_{i}^{\beta}\right] d y^{k} \wedge d y^{i} \wedge e^{\alpha} \\
& +\frac{1}{2}\left[\partial_{k} B_{i j}-\partial_{k} B_{i \alpha} \lambda_{j}^{\alpha}+B_{i \alpha} \partial_{k} \lambda_{j}^{\alpha}\right] d y^{i} \wedge d y^{j} \wedge d y^{k}
\end{aligned}
$$

As a 3 -form, we project $H$ on representations of SU(3) as we did for $d J$ :

$$
H=-\frac{3}{2} \operatorname{Im}\left(H_{1} \bar{\Omega}\right)+H_{4} \wedge J+H_{3}
$$

These components are computed with the same contractions used for $W$ 's:

$$
\begin{aligned}
h_{1}=H_{1}= & \left.-\frac{4 i}{3} \Omega\right\lrcorner H \\
= & \frac{1}{12} \epsilon^{i j k} V_{i}^{\alpha} V_{j}^{\beta} \partial_{k} B_{\alpha \beta} \\
& +\frac{i}{12} \epsilon^{i j k} V_{i}^{\alpha}\left[d B_{\alpha}-B_{\alpha \beta} d \lambda^{\beta}\right]_{j k} \\
& -\frac{1}{12} \epsilon^{i j k}\left[\partial_{k} B_{i j}-\partial_{k} B_{i \alpha} \lambda_{j}^{\alpha}+B_{i \alpha} \partial_{k} \lambda_{j}^{\alpha}\right] \\
H_{4}= & 2 J\lrcorner H \\
= & -\frac{1}{2} V^{k \alpha}\left[d B_{\alpha}-B_{\alpha \beta} d \lambda^{\beta}\right]_{j k} d y^{j}-\frac{1}{2} V^{k \alpha} \partial_{k} B_{\alpha \beta} e^{\beta} \\
= & h_{k}^{4} d z^{k}+\bar{h}_{k}^{4} d \bar{z}^{k} \\
h_{k}^{4}= & \frac{1}{4}\left\{V^{j \alpha}\left[d B_{\alpha}-B_{\alpha \beta} d \lambda^{\beta}\right]_{j k}-i V^{j \alpha} \partial_{j} B_{\alpha \beta} V_{k}^{\beta}\right\}
\end{aligned}
$$




$$
\begin{aligned}
H_{3}= & H+\frac{3}{2} \operatorname{Im}\left(H_{1} \bar{\Omega}\right)-H_{4} \wedge J \\
= & \frac{1}{4} V^{k \beta} V^{i \gamma}\left[\partial_{k} B_{i \alpha}-B_{\alpha \mu} \partial_{k} \lambda_{i}^{\mu}\right] e^{\alpha} \wedge e^{\beta} \wedge e^{\gamma} \\
& +\frac{1}{2}\left[\frac{5}{4} \partial_{k} B_{\alpha \beta}-V^{j \gamma} V_{k \alpha} \partial_{j} B_{\gamma \beta}-\frac{1}{2} V_{k}^{\gamma} V_{\alpha}^{j} \partial_{j} B_{\gamma \beta}\right] d y^{k} \wedge e^{\alpha} \wedge e^{\beta} \\
& +\frac{1}{8}\left[\partial_{k} B_{i j}-\partial_{i} B_{j \gamma} \lambda_{k}^{\gamma}-\partial_{i} \lambda_{j}^{\gamma} B_{k \gamma}\right]\left[V_{\beta}^{k} V_{\alpha}^{j} d y^{i}-V_{\beta}^{k} V_{\alpha}^{i} d y^{j}+V_{\alpha}^{i} V_{\beta}^{j} d y^{k}\right] \wedge e^{\alpha} \wedge e^{\beta} \\
& +\frac{1}{4}\left[\left(d B_{\alpha}\right)_{i k}-B_{\alpha \beta}\left(d \lambda^{\beta}\right)_{i k}\right]\left[\frac{3}{2} \delta_{j}^{k} \delta_{\gamma}^{\alpha}+V_{j}^{\alpha} V_{\gamma}^{k}-2 V^{k \alpha} V_{j \gamma}\right] d y^{i} \wedge d y^{j} \wedge e^{\gamma} \\
& +\frac{3}{8}\left[\partial_{k} B_{i j}-\partial_{i} B_{j \gamma} \lambda_{k}^{\gamma}-\partial_{i} \lambda_{j}^{\gamma} B_{k \gamma}\right] d y^{i} \wedge d y^{j} \wedge d y^{k} \\
& -\frac{1}{8} V_{j}^{\alpha} V_{k}^{\beta} \partial_{i} B_{\alpha \beta} d y^{i} \wedge d y^{j} \wedge d y^{k} \\
= & \frac{1}{2} h_{i j k}^{3} d z^{i} \wedge d \bar{z}^{j} \wedge d \bar{z}^{k}+\text { c. c. }
\end{aligned}
$$

In analogy with $w$ 's (see (4.6) - (4.9) ) we have introduced $h$ :

$$
\begin{aligned}
h_{i j}^{3}= & h_{i p q}^{3} \Omega^{p q}{ }_{j}=h_{\{i j\}^{0}}^{3}+\frac{1}{3} h_{t}^{3} g_{i j} \\
h_{\{i j\}^{0}}^{3}= & -\frac{1}{24} \epsilon^{p q k}\left[d B_{\alpha}\right]_{p q}\left[2 V_{k}^{\alpha} g_{i j}-3 V_{j}^{\alpha} g_{i k}-3 V_{i}^{\alpha} g_{j k}\right] \\
h_{t}^{3}= & -\frac{1}{8} \epsilon^{p q k}\left[d B_{\alpha}\right]_{p q} V_{k}^{\alpha} \\
& -\frac{i}{8} \epsilon^{p q k}\left[d B-\frac{1}{2}\left(d B_{\alpha} \wedge \lambda^{\alpha}+d \lambda^{\alpha} \wedge B_{\alpha}\right)\right]_{p q k}
\end{aligned}
$$

\section{References}

[1] S. Gurrieri, J. Louis, A. Micu and D. Waldram, "Mirror symmetry in generalized Calabi-Yau compactifications," Nucl. Phys. B 654, 61 (2003) arXiv:hep-th/0211102.

[2] C. Vafa, "Superstrings and topological strings at large N," J. Math. Phys. 42 (2001) 2798 arXiv:hep-th/0008142.

[3] A. Strominger, S. T. Yau and E. Zaslow, "Mirror symmetry is T-duality," Nucl. Phys. B 479 (1996) 243 arXiv:hep-th/9606040.

[4] N. Hitchin, "Generalized Calabi-Yau manifolds," arXiv:math.dg/0209099

[5] N. C. Leung, S. T. Yau and E. Zaslow, "From special Lagrangian to Hermitian-Yang-Mills via Fourier-Mukai transform," Adv. Theor. Math. Phys. 4 (2002) 1319 arXiv:math.dg/0005118.

[6] M. Marino, R. Minasian, G. W. Moore and A. Strominger, "Nonlinear instantons from supersymmetric p-branes," JHEP 0001 (2000) 005 arXiv:hep-th/9911206.

[7] M. R. Douglas, B. Fiol and C. Romelsberger, "Stability and BPS branes," arXiv:hep-th/0002037

[8] A. Strominger, "Superstrings With Torsion," Nucl. Phys. B 274 (1986) 253.

[9] J. P. Gauntlett, D. Martelli, S. Pakis and D. Waldram, "G-structures and wrapped NS5branes," arXiv:hep-th/0205050 
[10] G. L. Cardoso, G. Curio, G. Dall'Agata, D. Lust, P. Manousselis and G. Zoupanos, "NonKaehler string backgrounds and their five torsion classes," Nucl. Phys. B 652, 5 (2003) arXiv:hep-th/0211118.

[11] P. Bouwknegt, J. Evslin and V. Mathai, "T-duality: Topology change from H-flux," arXiv:hep-th/0306062

[12] S. F. Hassan, "SO(d,d) transformations of Ramond-Ramond fields and space-time spinors," Nucl. Phys. B 583 (2000) 431 arXiv:hep-th/9912236.

[13] D. D. Joyce, Compact manifolds with special holonomy, Oxford, 2000.

[14] P. Kaste, R. Minasian and A. Tomasiello, "Supersymmetric M-theory compactifications with fluxes on seven-manifolds and G-structures," JHEP 0307 (2003) 004 arXiv:hep-th/0303127.

[15] J. T. Liu and R. Minasian, "U-branes and T**3 fibrations," Nucl. Phys. B 510 (1998) 538 arXiv:hep-th/9707125.

[16] N. Hitchin, "Stable forms and special metrics," in Global differential geometry: the mathematical legacy of Alfred Gray, volume 288 of Contemp. Math. , pages 70-89. AMS, 2001. math.DG/0107101.

[17] S. Chiossi and S. Salamon, "The intrinsic torsion of $\mathrm{SU}(3)$ and $\mathrm{G}_{2}$ structures," Proc. conf. Differential Geometry Valencia 2001, math.DG/0202282

[18] K. Becker, M. Becker, P. S. Green, K. Dasgupta and E. Sharpe, "Compactifications of heterotic strings on non-Kaehler complex manifolds. II," arXiv:hep-th/0310058.

[19] R. Rohm and E. Witten, Annals Phys. 170 (1986) 454.

[20] M. Grana and J. Polchinski, "Gauge / gravity duals with holomorphic dilaton," Phys. Rev. D 65 (2002) 126005 arXiv:hep-th/0106014.

[21] P. Kaste, R. Minasian, M. Petrini and A. Tomasiello, "Nontrivial RR two-form field strength and SU(3)-structure," arXiv:hep-th/0301063

[22] A. Kapustin, "Topological strings on noncommutative manifolds," arXiv:hep-th/0310057

[23] A. Kapustin and D. Orlov, "Vertex algebras, mirror symmetry, and D-branes: The case of complex tori," Commun. Math. Phys. 233 (2003) 79 arXiv:hep-th/0010293. 